discharge. Only $28 \%$ have access to a psychologist. In-hospital stroke alerts $(p=0.014)$, access to thrombectomy $(p=0.016)$, access to stroke unit care $(\mathrm{p}=0.027)$, and routine referral to stroke foundation $(p=0.049)$ were more common in urban areas.

Conclusion The results of this organisational survey indicates that stroke care provision has improved since the last audit in 2009, but important gaps remain. These results will help services focus on specific areas for improvement, some of which such as pre-hospital alerts should be relatively easy to address.

\section{SILENT MULTILEVEL VERTEBRAL FRACTURES IN A SEVERE CASE OF GLYCINE RECEPTOR ANTIBODY- POSITIVE PROGRESSIVE ENCEPHALOMYELITIS WITH RIGIDITY AND MYOCLONUS (PERM)}

Myintzu Min*, Han Liu*, Paul Silberstein. Royal North Shore Hospital, Sydney, NSW, Australia

\subsection{6/jnnp-2019-anzan.92}

Introduction Autoimmune encephalitides are a heterogenous and potentially devastating group of disorders. Antibodies to glycine receptor (GlyR) are rare and increasingly reported in patients with progressive encephalomyelitis with rigidity and myoclonus (PERM). PERM patients develop early brainstem and autonomic dysfunction, and if untreated, can be fatal. We aim to discuss the complications and treatments in this PERM case and review the literature on management of PERM.

Methods We report a case of PERM currently undergoing treatment in our hospital.

Results Mr GT is a 46-year-old male who presented with a prodromal phase of altered sensation and acute cerebellar signs. He rapidly deteriorated with bulbar dysfunction and developed generalised muscle rigidity and hyperreflexia. Infective work-up, cerebrospinal fluid analysis, magnetic resonance imaging of brain and spine were initially normal. He was started on first line immunosuppressants for a clinical diagnosis of autoimmune encephalitis. Despite sedation in intensive care, generalised myoclonus and truncal extensor spasms were severe and presented on-going management difficulties. A computed tomography scan of his abdomen to exclude malignancy revealed incidental findings of vertebral fractures at multiple thoracic and lumbar levels. $\mathrm{He}$ was subsequently administered rituximab and demonstrated some signs of recovery. GlyR antibody positive results from Oxford University Hospital consequently confirmed his diagnosis.

Conclusion This is the fourth reported GlyR antibody-positive PERM case in Australia and the first description to our knowledge with intractable rigidity and myoclonus leading to vertebral fractures. Awareness and screening for this complication may assist in management of patients with PERM.

\section{A PRESENTATION RESEMBLING TRANSIENT GLOBAL AMNESIA WITH UNDERLYING TAKO-TSUBO CARDIOMYOPATHY AND MULTIFOCAL ISCHAEMIC STROKE (WITH RESTRICTED DIFFUSION IN THE LEFT MESIOTEMPORAL LOBE)}

Kristen Lefever, Chloe Tyson, Lisa Shelley, Daniel Schweitzer, Andrew Swayne*. Centre for Neurosciences, Mater Hospital, Brisbane, QLD, Australia

10.1136/jnnp-2019-anzan.93
Introduction We present a case of a 65 year-old female with a presentation resembling transient global amnesia (TGA) with investigations revealing a Tako-tsubo cardiomoyopathy and multifocal punctate areas of restricted diffusion consistent with ischaemic stroke.

Methods Single case report.

Results A 65 year-old female presented altered behaviour with anterograde amnesia consistent with an episode of TGA. There was no clearly identifiable stressor to provoke this episode. The TGA-like symptoms persistent for 12 hours before the patient made a full recovery.

During the presentation the patient complained of chest pain with elevated troponin. Cardiac investigation was notable for mild left ventricular dilation with mid- and apical hypokinesis on transthoracic echocardiogram and a normal coronary angiogram consistent with Tako-tsubo cardiomyopathy. Magnetic resonance imaging of the brain revealed multifocal infarcts including punctate foci of restricted diffusion within the left mesiotemporal lobe.

Conclusion This case is notable as it is rare to have a combined presentation with TGA-type symptoms with Tako-tsubo cardiomyopathy and multifocal ischaemia stroke. ${ }^{1}$ This is the first clinical case presentation involving the aforementioned triad and mesiotemporal lobe restricted diffusion. We hypothesise that the Tako-tsubo cardiomyopathy acted as the triggering event and the mesiotemporal lobe involvement caused the TGA-like symptoms thereby accounting for the clinical presentation and investigation findings. As mesiotemporal/hippocampal restricted diffusion may be seen with transient global amnesia, ${ }^{2}$ this case is of special interest mechanistically although we cannot exclude the alternative hypothesis that the episode of TGA resulted in the Takotsubo cardiomyopathy which subsequently caused the cardioembolic shower.

\section{REFERENCES}

1. Stollberger C, DeCillia N, Finsterer J. 'Tako-tsubo cardiomyopathy with transient global amnesia and cerebellar embolic stroke triggered by existential fear.' Neurol Neurochir Pol 2018; 52(3):394-396.

2. Enzinger C, Thimary F, Kapeller P, Ropele S, Schmidt R, Ebner F, Fazekas F. 'Transient global amnesia: diffusion-weighted imaging lesions and cerebrovascular disease.' Stroke 2008:39(8):2219-2225.

\section{HAEMORRHAGIC SPINAL CORD INFARCT - A RARE COMPLICATION OF SYMPATHOMIMETIC AMINE TOXICITY}

Ellen L Wall*, Jerome A Leow, Jonathan Ho, Yun T Hwang. Central Coast Local Health District, Gosford, NSW, Australia

10.1136/jnnp-2019-anzan.94

Introduction Sympathomimetic amines are recreational substances, available illegally as amphetamine derivatives (eg, 'ecstasy' and 'speed'). Ingestion can lead to significant medical complications such as hyperthermia, tachyarrhythmia, seizures and strokes, attributed to catecholamine surge and sympathetic overstimulation.

Method We report an unusual case of sympathomimetic amine ingestion manifesting as hypertension followed by acute onset flaccid paralysis of lower limbs bilaterally and T11 level anaesthesia in a 64 year old woman secondary to haemorrhagic cord infarct.

Results An MRI spine showed features compatible with spinal cord infarction from thoracic level $6 / 7$ to conus associated 
with cord haemorrhage. There was no evidence of vascular malformations on imaging and the screen for inflammatory myelitides was negative. A urine drug screen tested positive for sympathomimetic amines and the patient acknowledged ingesting a pill of 'unknown identity'.

Conclusion This case highlights a previously unreported complication of recreational sympathomimetics associated with significant patient morbidity. Unfortunately, this woman failed to make significant improvements during admission with ongoing severe motor and sensory deficits of her lower limbs.

\section{ENDOVASCULAR CLOT RETRIEVAL BEYOND 24 HOURS FOR TOP OF THE CAROTID OCCLUSION}

${ }^{1}$ Paul Kopanidis*, 'Shaun Zhai, ${ }^{2}$ Shivendra Lalloo, 1,3 Ronak Patel, 1,3Yash Gawarikar. ${ }^{1}$ Neurology, Calvary Public Hospital, Bruce, ACT, Australia; ${ }^{2}$ Radiology, The Canberra Hospital, Canberra, ACT, Australia; ${ }^{3}$ Medical School, Australian National University, Canberra, ACT, Australia

\subsection{6/jnnp-2019-anzan.95}

Introduction Sub-clinoid proximal occlusion is defined by internal carotid artery (ICA) occlusion with intact Circle of Willis flow. We hypothesise that such cases of large vessel occlusion provide collateral blood flow to preserve the ischaemic penumbra and may benefit from endovascular clot retrieval (ECR) beyond 24 hours.

Method We retrospectively searched the stroke database from 2018 at Calvary Hospital, Canberra, Australia for ECR cases performed beyond 24 hours from symptom onset.

Results Two patients were identified from the registry data.

64-year-old man awoke with left hemiparesis and was last seen well 9.5 hours prior. ECR for ICA occlusion was not initially performed due to rapidly improving National Institute of Health Stroke Scale (NIHSS) of three to zero. ECR was later performed at 38.5 hours for clinical deterioration. Stroke aetiology was atrial fibrillation. At 90-day NIHSS and modified Rankin Scale (mRS) were three.

75 -year-old man awoke with left hemiparesis and was last seen well 10 hours prior. Baseline NIHSS was four. Off-label thrombolysis was administered based on salvageable penumbra on CTP, however ECR for ICA occlusion was not performed as neurointervention was unavailable. After 24 hours his NIHSS score improved to one but hemispheric hypoperfusion persisted on CTP. At 36 hours he underwent ECR with carotid stenting. Stroke aetiology was large-vessel atherosclerosis. At 90 days his NIHSS and mRS were zero.

Conclusion Acute sub-clinoid proximal carotid occlusion requires tissue viability assessment with imaging to guide decision of ECR beyond 24 hours and may be of benefit.

\section{SEVERE DYSAUTONOMIA IN NMDAR ENCEPHALITIS}

${ }^{1}$ Alana Donaldson*, ${ }^{1}$ Abhay Venkat*, ${ }^{1,2}$ Shaun Zhai, ${ }^{1,2}$ Ronak Patel, ${ }^{1,2}$ Yash Gawarikar. ${ }^{1}$ Calvary Public Hospital Bruce, Canberra, ACT, Australia; ${ }^{2}$ Australian National University, Canberra, ACT, Australia

10.1136/jnnp-2019-anzan.96

Introduction Anti-N-methyl-D-aspartate receptor (NMDAR) encephalitis is one of the more common forms of autoimmune encephalitis, predominantly affecting children and women of the child-bearing age. It is characterised by memory deficit, behavioural disturbance and seizures. Dysautonomia is recognised as a feature but rarely the first symptom of the condition. ${ }^{1}$ Here we present a case of severe dysautonomia preceding the diagnosis of NMDAR encephalitis.

Methods We conducted a retrospective review of the admissions to the neurology ward at the Calvary Hospital, Canberra in 2018 to identify patients diagnosed with NMDAR encephalitis.

Results One patient was identified from the registry data. A 37-year-old woman presented with a week-long history of symptomatic orthostatic hypotension. Her supine systolic blood pressure was $110 \mathrm{mmHg}$ with a $46 \mathrm{mmHg}$ postural drop. Over the first week of hospitalisation, she became increasingly disoriented and erratic in behaviour with fluctuating levels of consciousness requiring intensive unit care. Her CSF demonstrated lymphocytic pleocytosis and NMDAR antibodies were detected in both CSF and serum. She was treated with IVIG, IV steroids and subsequently Rituximab. A pelvic teratoma was found and removed. Her symptomatology including dysautonomia improved substantially by the end of her six-week hospital admission. Her modified Rankin Scale was zero at three months.

Conclusion Autonomic dysfunction is not a common feature of autoimmune encephalitides. Our case highlighted the possibility that dysautonomia can be the initiating symptom of this disease entity. Physician awareness is important in the early recognition and treatment of this condition.

\section{REFERENCE}

1. Titulaer MJ, McCracken L, Gabilondo I, Armangue T, Glaser C, lizuka T, Honig LS, Benseler SM, Kawachi I, Martinez-Hernandez E, et al. Treatment and prognostic factors for long-term outcome in patients with anti-NMDA receptor encephalitis: an observational cohort study. Lancet Neurol 2013;12 (2):157-165

\section{THE BENEFITS OF A CLINICAL TRIAGE TOOL IN ACUTE STROKE}

Khaled Alanati*, Sean Byrnes, James Evans, David Campbell, Ellen Wall. Department of Neuroscience, Gosford Hospital, Gosford, NSW, Australia

\subsection{6/jnnp-2019-anzan.97}

Introduction The advances in management of ischemic stroke and the extended treatment window have greatly increased the demand for acute stroke assessment. There is a need for fast and accurate triage both to identify candidates for acute stroke assessment and to minimize cost and time expended on unnecessary assessments and investigations.

We examined the potential benefit of a web-based triage tool designed to be used in the emergency department to identify patients suitable for hyperacute treatment based on the current standard of care.

Methods We performed a retrospective analysis of Electronic medical records of 235 patients, reviewed by the neurology team at Gosford Hospital for the consideration of hyperacute treatment over six months in 2017. Each patient's time of onset of symptoms, premorbid function and presenting deficit, as well as the type of treatment received, were collected. By entering those data to our new triage system, we estimated the number of clinical reviews and advanced imaging that could have been avoided. 